\title{
Milk metabolites and their genetic variability
}

\author{
D. Wittenburg, ${ }^{* 1}$ N. Melzer, ${ }^{*}$ L. Willmitzer,† J. Lisec,† U. Kesting,ł N. Reinsch, ${ }^{*}$ and D. Repsilber ${ }^{* 1}$ \\ *Institute for Genetics and Biometry, Unit Biomathematics and Bioinformatics, Leibniz Institute for Farm Animal Biology, \\ 18196 Dummerstorf, Germany \\ †Max Planck Institute for Molecular Plant Physiology, 14476 Potsdam-Golm, Germany \\ łLandeskontrollverband für Leistungs- und Qualitätsprüfung Mecklenburg-Vorpommern e.V. (LKV), 18273 Güstrow, Germany
}

\section{ABSTRACT}

The composition of milk is crucial to evaluate milk performance and quality measures. Milk components partly contribute to breeding scores, and they can be assessed to judge metabolic and energy status of the cow as well as to serve as predictive markers for diseases. In addition to the milk composition measures (e.g., fat, protein, lactose) traditionally recorded during milk performance test via infrared spectroscopy, novel techniques, such as gas chromatography-mass spectrometry, allow for a further analysis of milk into its metabolic components. Gas chromatography-mass spectrometry is suitable for measuring several hundred metabolites with high throughput, and thus it is applicable to study sources of genetic and nongenetic variation of milk metabolites in dairy cows. Heritability and mode of inheritance of metabolite measurements were studied in a linear mixed model approach including expected (pedigree) and realized (genomic) relationship between animals. The genetic variability of 190 milk metabolite intensities was analyzed from 1,295 cows held on 18 farms in Mecklenburg-Western Pomerania, Germany. Besides extensive pedigree information, genotypic data comprising 37,180 single nucleotide polymorphism markers were available. Goodness of fit and significance of genetic variance components based on likelihood ratio tests were investigated with a full model, including marker- and pedigree-based genetic effects. Broad-sense heritability varied from zero to 0.699 , with a median of 0.125 . Significant additive genetic variance was observed for highly heritable metabolites, but dominance variance was not significantly present. As some metabolites are particularly favorable for human nutrition, for instance, future research should address the identification of locus-specific genetic effects and investigate metabolites as the molecular basis of traditional milk performance test traits.

\footnotetext{
Received April 18, 2012.

Accepted December 13, 2012.

${ }^{1}$ Corresponding authors: wittenburg@fbn-dummerstorf.de and repsilber@fbn-dummerstorf.de
}

Key words: metabolome, genomic relationship, single nucleotide polymorphism, heritability

\section{INTRODUCTION}

In dairy cattle, a multitude of milk components are recorded during milk performance tests. Besides monitoring performance traits (e.g., fat or protein content), it is especially important to control udder health by means of indicator traits, such as cell count. Other milk composition traits, for instance, FA levels or acetone, are also involved in indicating management status of the cow. Most of these traits can be measured with infrared spectroscopy. Infrared spectroscopy has been extended to a more detailed analysis of milk components; for example, protein content has been further resolved into its components, such as $\kappa-\mathrm{CN}$ and $\beta-\mathrm{LG}$ (Rutten et al., 2011). The same technique is used to measure the FA composition of milk (Soyeurt et al., 2006). Although infrared spectroscopy is suitable for population-wide animal recording, novel techniques allow for a further analysis of milk (Töpel, 2004). Coupling GC-MS breaks milk down into its metabolic intermediates. Besides GC-MS, other technical processes (e.g., nuclear magnetic resonance spectroscopy; Nicholson et al., 1999) are available, which differ from GC-MS in terms of quantity, reproducibility, and sensitivity in matter determination. Klein et al. (2010) have applied GC-MS to milk samples and obtained a few metabolites for further investigation; in plants, however, GC-MS has been shown to be suitable for identification and relative quantification of several hundred metabolites in high throughput (40 min per sample; Lisec et al., 2006). Thus, GC-MS is applicable to study sources of genetic and nongenetic variation of milk metabolites in dairy cows. The milk metabolome is a snapshot of the metabolic state of a cow; thus, metabolites may primarily help to explore metabolic (production) diseases, such as ketosis, milk fever, rumen acidosis, or fatty liver syndrome (Littledike et al., 1981; Goff and Horst, 1997), and to infer the risk of a disease. For instance, 3-hydroxybutanoic acid is typically used as a biomarker for ketosis (Geishauser et al., 2000), and a related study 
found a direct relationship between ketosis and the ratio of nuclear magnetic resonance spectroscopy-derived milk components from glycerophosphocholine to phosphocholine (Klein et al., 2012). Furthermore, some milk components may be of particular interest; for example, bovine milk oligosaccharides (BMO) resemble human milk oligosaccharides in structure and have a similar protective role on infants' intestines and immune system (Zivkovic and Barile, 2011) and may be a target for functional food production. Thus, milk metabolites may not only be used as biomarkers, they can also generally be seen as novel milk traits from which other production or fitness traits may be deduced and whose genetic background should be elucidated. Then, it is necessary to quantify the extent to which the observed variability of metabolic profiles is due to either genetic variation or environmental or temporal variation.

The primary interest of this study was to estimate genetic parameters and explore mode of inheritance of milk metabolites which were obtained from milk samples collected during performance testing. Toward these objectives, genetic variability of metabolite measurements was investigated in a linear mixed model approach including expected (pedigree) and realized (genomic) relationship between animals for a large sample of dairy cows. The significance of genetic variance components was tested and the ability of milk metabolites to predict genetic values for nonphenotyped animals was studied to verify their meaning for breeding purposes. The heritable effect on chemically related metabolites as well as functionally related metabolites is discussed with respect to selected groups and pathways.

\section{MATERIALS AND METHODS}

\section{Milk Samples and Metabolite Data}

This field study was designed to gather samples under the same conditions as performance testing. Milk samples were collected from 1,344 Holstein cows held on 18 dairy farms in Mecklenburg-Western Pomerania (in the northeast of Germany) from May to November 2009. The cows were sampled between d 21 and 120 of their first lactation. Only first-lactation cows were selected to avoid variation due to parity and effects of selection due to culling of cows with low milk yield. As this project cooperated with commercial dairy farms only, a logistical challenge existed to separate samples of preselected cows that lay in the required lactation period. For this purpose, the LKV (Association for Quality Inspection, Güstrow, Germany) had access to a farm-specific animal list published and updated weekly on a website. The quality inspector drew 2 samples from the aliquot of the cow's daily milk yield - one according to the usual procedure for milk performance tests, and the other drawn for use in the experiment. Preservatives (natrium acid) were added to the milk samples. The additional samples were transported in separate boxes to the LKV, and analyzed via infrared spectroscopy (Foss, Hillerød, Denmark). Afterward, the remaining milk was aliquoted into 2-mL tubes (Eppendorf, Germany) and frozen using liquid nitrogen until sample collection was finished (39 collection dates). Samples were deep-frozen within a few hours after being collected on a regular test day. All samples (one tube per cow) were sent to the laboratory at the Max Planck Institute for Molecular Plant Physiology (MPIMP; Potsdam, Germany) to measure the metabolite profiles. Twelve animals were later removed from the data set due to an invalid milk measurement (outside the desired lactation interval or because of decreased $\mathrm{pH}$ value).

Profiles for the hydrophilic fraction of metabolites were obtained from GC-MS according to Lisec et al. (2006) with minor adjustments [no ball milling, 1.1-mL total extraction volume; $100 \mu \mathrm{L}$ of milk sample $+1 \mathrm{~mL}$ of $\mathrm{MeOH}: \mathrm{CHCl}_{3}: \mathrm{H}_{2} \mathrm{O} ; 10: 90 \mathrm{~N}$-methyl-N-trimethylsilyltrifluoroacetamide (MSTFA) derivatization]. Sample data were unbalanced in terms of farm, sample date, and half-sib families. For example, a sire had, on average, 6.6 daughters, ranging from 1 to 106. Thus, for GC-MS application, a specific randomized design based on a Latin square was developed, which was as balanced as possible with respect to the factors mentioned above (Melzer et al., 2010). Because of laboratory restrictions, the final design was slightly modified. The laboratory delivered molecule spectra, measured in 47 batches, in which molecule retention time (GC step), the mass:charge ratios, and the corresponding relative intensities of molecule fragments (MS step) were recorded for each sample. These spectra were further processed with the $\mathrm{R}$ package TargetSearch version 1.10 (Cuadros-Inostroza et al., 2009; R Development Core Team, 2011). The retention time of each molecule was converted into a retention index based on the retention time standards of FA methyl esters added to the sample in the GC step. Both retention index and molecule spectrum were used to annotate each molecule obtained from the MS step. Molecule spectra from narrow time windows $(0.5 \mathrm{~s})$, which showed highly correlated intensity values (correlation >0.95) over all samples, were combined to build a metabolite spectrum. Median values of these mass spectra were then compared with reference spectra in a database (Golm Metabolome Database, GMD; http://gmd. mpimp-golm.mpg.de/search.aspx). The assignment of a metabolite spectrum to the reference was accepted in case that the similarity score $(\in[0 ; 1,000])$ between them was >500; otherwise, the metabolite was labeled 




Figure 1. Metabolite spectrum of 1,6-anhydro- $\beta$-glucose; the spectrum above the null line was obtained as median of peak intensities of all cows along the mass:charge ratio $[\mathrm{m} / \mathrm{z}]$ of molecule fragments, and the matching reference spectrum below the null line was obtained from a reference substance. The similarity score between metabolite spectrum and reference was 639 .

as unknown. As an example, Figure 1 shows a median metabolite spectrum mapped to the corresponding reference. The intensity at the largest peak was taken as individual observation; metabolites with more than $20 \%$ missing values were omitted. Using these criteria, 187 identified and 3 unknown metabolites could be measured. The database mentioned above also allowed for assorting the metabolites with respect to their chemical groups. This structure was also used to give an overview of results of investigations.

\section{Marker Data and Pedigree Structure}

Blood samples were taken from all cows on a multiple-day tour with a veterinarian. Purification of DNA from blood was carried out with a commercially available kit (NucleoSpin Blood L, Macherey-Nagel, Düren, Germany). The concentration of DNA was controlled with NanoDrop 1000 Spectrophotometer (Thermo Fisher Scientific, Wilmington, MA), ranging from 50 to $100 \mathrm{ng} / \mu \mathrm{L}$. The laboratory at the Helmholtz Zentrum München, Germany, determined SNP genotypes using the Illumina BovineSNP50 BeadChip (Illumina Inc., San Diego, CA). Out of 54,001 SNP on the chip, 48,713 SNP with known position were identified via BLAST analysis (Altschul et al., 1990) based on the SNP annotation Btau4.2 (The Bovine Genome Sequencing and Analysis Consortium, 2009). Then, 27 animals were skipped for having more than $10 \%$ missing SNP genotypes. Standard quality checks were applied to the SNP data (Ziegler et al., 2008); loci that were not in Hardy-Weinberg equilibrium and loci with minor allele frequency $<5 \%$, or with more than $10 \%$ missing genotypes, were omitted. In total, $m$ $=37,180 \mathrm{SNP}$ were retained. The rarely missing genotypes $(0.5 \%)$ of these SNP were imputed by randomly drawing missing alleles according to observed allele frequencies. Furthermore, 10 additional animals were excluded because deviations between the realized (genomic) and expected (pedigree) relationship indicated Mendelian inconsistencies. Consistencies were assumed when $90 \%$ of the absolute differences between relationship coefficients were smaller than 0.2 (Calus et al., 2011). Thus, $n=1,295$ cows with proper genotypes and phenotypes remained for analyses. Cows were descendants of 192 sires, but 22 cows had unknown sires; the pedigree included 23,819 animals with up to 11 generations backward (obtained from the data center, VIT, Verden, Germany).

\section{Statistical Model}

The genetic variability of milk metabolites was studied taking the genomic BLUP (GBLUP) approach (Habier et al., 2007; VanRaden, 2008), for which a robust behavior in terms of accuracy of genetic value prediction was shown for various traits (Daetwyler et al., 2010). As metabolites were observed and analyzed on the level of genotyped cows, both additive and dominance genetic effects could be considered; this required a proper extension of the GBLUP approach to include dominance. 
The raw metabolite intensities were $\log _{2}$-transformed to approach normality and then analyzed in a series of univariate analyses, one per metabolite. The vector $\mathbf{y}=$ $\left(y_{1}, \ldots, y_{n}\right)^{\prime}$ consisted of $\log _{2}$ intensities for animals $i=$ $1, \ldots, n$. Genetic variance captured by the markers, as well as polygenic effects, which cover residual additive genetic variation, were considered. The following linear mixed model was fitted to the data (bold indicating matrices and vectors):

$$
\mathbf{y}=\mathbf{X b}+\mathbf{Z}_{a} \mathbf{u}_{a}+\mathbf{Z}_{d} \mathbf{u}_{d}+\mathbf{Z}_{p} \mathbf{u}_{p}+\mathbf{e} .
$$

The residuals were assumed to be independently and normally distributed, $e_{i} \sim N\left(0, \sigma_{e}^{2}\right)$ for $i=1, \ldots, n$. The vector $\mathbf{b}$ included systematic effects on the milk metabolites and considered fixed effects of farm $\times$ sampling date (63 levels), metabolite measurement day (batch effect, 47 levels) and, to account for varying metabolism in different stages of lactation, linear and quadratic regression on lactation day with suitable entries in the design matrix $\mathbf{X}$. The design matrices $\mathbf{Z}_{a}$ for the additive genetic values $\left(\mathbf{u}_{a}\right)$ and $\mathbf{Z}_{d}$ for the dominance genetic values $\left(\mathbf{u}_{d}\right)$ were identity matrices of proper size because each individual was phenotyped and genotyped. The polygenic effect $\mathbf{u}_{p} \sim N\left(\mathbf{0}, \mathbf{A} \sigma_{p}^{2}\right)$ with design matrix $\mathbf{Z}_{p}$ involved the numerator relationship matrix $\mathbf{A}$ obtained from pedigree information, and it was assumed to be uncorrelated with genetic effects explained by markers. The direct genetic value $D G V_{i}$ of animal $i$ was defined as the sum of effect-specific genetic values; that is, $D G V_{i}=u_{a, i}+u_{d, i}+u_{p, i}$.

Next, the covariance matrices of the effect-specific genetic values were inferred. The genetic value of type $s \in\{a, d\}$ was defined as the sum of genetic effects depending on the individual SNP genotypes, that is, $\mathbf{u}_{s}=$ $\mathbf{M}_{s} \mathbf{g}_{s}$. The $(n \times m)$ matrix $\mathbf{M}_{s}$ with columns $\mathbf{M}_{s, 1}, \ldots$, $\mathbf{M}_{s, m}$ included orthogonalized genotype coefficients for the genetic effects $\mathbf{g}_{s}=\left(g_{s, 1}, \ldots, g_{s, m}\right)^{\prime}$ over the whole genome. This way, the additive genetic values $\mathbf{M}_{a, j} g_{a, j}$ and the dominance deviations $\mathbf{M}_{d, j} g_{d, j}$ were uncorrelated at locus $j \in\{1, \ldots, m\}$. Let 1 and 2 denote the SNP alleles at locus $j$, where 2 means the more frequent allele with frequency $p_{j}$. Depending on the observed marker genotypes, the entries in $\mathbf{M}_{s}$ were obtained according to Zeng et al. (2005) as follows:

$$
M_{a, i, j}=\left\{\begin{array}{cc}
-2 p_{j} & \text { genotype } 11 \\
1-2 p_{j} & \text { genotype } 12 \\
2\left(1-p_{j}\right) & \text { genotype } 22
\end{array}\right.
$$

$$
\text { and } M_{d, i, j}=\left\{\begin{array}{cc}
-2 p_{j}^{2} & \text { genotype 11 } \\
2 p_{j}\left(1-p_{j}\right) & \text { genotype 12. } \\
-2\left(1-p_{j}\right)^{2} & \text { genotype 22 }
\end{array}\right.
$$

These matrices were additionally standardized column by column:

$$
\begin{gathered}
\mathbf{M}_{a, j} \mapsto \frac{\mathbf{M}_{a, j}}{\sqrt{2 p_{j}\left(1-p_{j}\right)}} \\
\text { and } \mathbf{M}_{d, j} \mapsto \frac{\mathbf{M}_{d, j}}{2 p_{j}\left(1-p_{j}\right)}
\end{gathered}
$$

Thus, only correlations between SNP were of prime interest, meaning that SNP with rare alleles were as important as SNP with medium allele frequency for statistical investigations. If it was assumed that $g_{s, 1}$, $\ldots, g_{s, m}$ are independent and identically distributed and each locus contributes to the genetic variation with $\operatorname{Var}\left(g_{s, j}\right)=\sigma_{s}^{2}$, then the covariance matrix of the genetic values could be derived as

$$
\operatorname{Var}\left(\mathbf{u}_{s}\right)=\mathbf{M}_{s} \mathbf{M}_{s}^{\prime} \sigma_{s}^{2}=: \mathbf{G}_{s} m \sigma_{s}^{2} .
$$

The genomic relationship matrix $\mathbf{G}_{a}=\frac{1}{m} \mathbf{M}_{a} \mathbf{M}_{a}^{\prime}$ corresponded exactly to the $\mathbf{G}$ matrix of VanRaden (2008; version 2) and, in case of dominance, $\mathbf{G}_{d}=\frac{1}{m} \mathbf{M}_{d} \mathbf{M}_{d}^{\prime}$ included the correlation of dominance deviations. Thus, in the model above, it was assumed that $\mathbf{u}_{s} \sim N\left(\mathbf{0}, \mathbf{G}_{s} m \sigma_{s}^{2}\right)$ for $s \in\{a, d\}$.

Let $\sigma_{A}^{2}=m \sigma_{a}^{2}$ denote the additive genetic variance captured by the markers and, analogously, $\sigma_{D}^{2}=m \sigma_{d}^{2}$ denotes the dominance variance. Narrow-sense heritability $\left(\boldsymbol{h}^{2}\right)$ and broad-sense heritability $\left(\boldsymbol{H}^{2}\right)$ were determined as follows:

$$
\begin{gathered}
h^{2}=\frac{\sigma_{A}^{2}+\sigma_{p}^{2}}{\sigma_{A}^{2}+\sigma_{p}^{2}+\sigma_{D}^{2}+\sigma_{e}^{2}} \\
\text { and } H^{2}=\frac{\sigma_{A}^{2}+\sigma_{p}^{2}+\sigma_{D}^{2}}{\sigma_{A}^{2}+\sigma_{p}^{2}+\sigma_{D}^{2}+\sigma_{e}^{2}} .
\end{gathered}
$$


Based on the full model, including marker- and pedigree-based genetic effects, goodness of fit was evaluated by metabolite-wise visual inspection of studentized residuals.

\section{Testing Significance of Genetic Variance Components}

Assuming independent loci, and under the given parameterization of genotype coefficients, the additive genomic relationship matrix $\mathbf{G}_{a}=\frac{1}{m} \mathbf{M}_{a} \mathbf{M}_{a}^{\prime}$ approximates the numerator relationship matrix $\mathbf{A}$, where the quality of approximation depends on the number of loci $m$ (Habier et al., 2007). Because of the finite number of loci and their dependence due to linkage, it makes sense to additionally account for genetic background in the model. To evaluate the significant presence of the polygenic variation, the null hypothesis $H_{0}: \sigma_{p}^{2}=0$ versus the alternative hypothesis $H_{A}: \sigma_{p}^{2}>0$ was tested via residual likelihood ratio test. This led to a nonstandard testing problem because the parameter to be tested lay at the boundary of its parameter space under $H_{0}$. The asymptotic null distribution of the test statistic is a $\frac{1}{2}: \frac{1}{2}$ mixture of $\chi^{2}$ distribution with 1 degree of freedom and point mass $\delta_{0}$ at zero (Self and Liang, 1987; case 5). The significance of any additive genetic variation (i.e., both marker- and pedigree-based genetic variability) was also validated. Under the corresponding null hypothesis $H_{0}: \sigma_{A}^{2}=0$ and $\sigma_{p}^{2}=0$, the distribution of the residual likelihood ratio test statistic asymptotically follows a $\frac{1}{4}: \frac{1}{2}: \frac{1}{4}$ mixture of $\delta_{0}$ and $\chi^{2}$ distributions with 1 and 2 degrees of freedom (Self and Liang, 1987, case 9). Furthermore, the significance of the dominance variation was analyzed with $H_{0}: \sigma_{D}^{2}=0$. Again, the residual likelihood ratio test null distribution being $\frac{1}{2} \delta_{0}+\frac{1}{2} \chi_{1}^{2}$ was involved.

In metabolite-wise investigations, the type-I error was $5 \%$; the $P$-values were adjusted to control the false discovery rate at $5 \%$ over all metabolites (Benjamini and Hochberg, 1995). Unless explicitly mentioned, all results presented below are based on false discovery rate-corrected data.

\section{Testing Significance of Fixed Effects}

For evaluation of metabolite measurements varying over the different stages of lactation, the significance of the regression coefficients concerning lactation day was tested by an $F$-test with adjusted denominator degrees of freedom (Kenward and Roger, 1997). In the software applied (see below), linear and quadratic regression on lactation day were realized with orthogonal polynomials of first and second degree. Therein, the $F$-test considered the null hypothesis of both regression coefficients being zero.

To verify which order of regression on lactation day was actually appropriate, nonparametric regression was applied to precorrected metabolite data and the equivalent number of parameters $\left(p_{e}\right)$ was determined (Ruppert et al., 2003). Then, a $\left(p_{e}-1\right)$-degree polynomial was suggested to fit the data. Precorrection was obtained via linear mixed model including fixed effects of farm $\times$ sampling date, measurement day, and a random sire effect to account for similarities among half-sibs. The method of choice was a penalized B-splines approach based on 20 knots (every fifth day).

The significance of farm $\times$ sampling-date effect and metabolite measurement-day effect were also tested by an $F$-test.

\section{Predictive Ability}

The predicted genetic value of an animal is an essential figure in breeding applications. The accuracy of predicting genetic values of nonphenotyped animals with milk metabolites observed in a training data set depends on heritability and relationship between animals. To study predictive ability of the GBLUP approach for metabolites in general, the accuracy of predicting genetic values, as well as predicting metabolite intensities, was evaluated. Predictive ability was assessed using a leave-one-out cross-validation (Hastie et al., 2009). In a successive manner, each single metabolite measurement was omitted from the training data and the cow's genetic value was predicted based on the present covariance structure. The estimates of fixed effects were also considered for predicting the metabolite intensity; after this was done for each animal, the empirical correlation $(\rho)$ between observed metabolite measurements and predicted genetic values served as a measure of accuracy of genetic value prediction. Accuracy of phenotype prediction was determined as empirical correlation between observed and predicted metabolite measurements. Because of the computational effort of fitting the full model, a reduced model $\left(\sigma_{p}^{2}=0\right)$ was applied to selected metabolites. Three highly heritable metabolites were chosen from the chemical groups of sugars and alcohols; then, the metabolite with highest heritability (based on the full model) and another arbitrarily chosen metabolite with medium heritability were investigated. 


\section{Software}

Quality checks on the SNP genotypes and set-up of genomic relationship matrices were implemented in Fortran 95; the imputation of rarely missing genotypes was performed using Beagle 3.2 (Browning and Browning, 2007). The estimation of systematic and genetic effects and the REML estimation of variance components were carried out with ASReml 3.0 (Gilmour et al., 2008). Model fit, likelihood ratio testing (based on the output of ASReml) and the cross validation were carried out in $\mathrm{R}$ ( $\mathrm{R}$ Development Core Team, 2011). The B-spline approach was ready implemented in the $\mathrm{R}$ function smooth.spline.

\section{RESULTS}

\section{Model Fit}

The raw metabolite intensities were heavily leftskewed (Supplemental Figure S1, available online at http://www.journalofdairyscience.org/). For most metabolites, the distribution of the $\log _{2}$-transformed metabolite measurements had a symmetric shape and approximately followed a normal distribution; for example, the histogram of 3-hydroxybutanoic acid is shown in Figure 2a. For a few metabolites, the fit at the tails was slightly insufficient due to increased frequency of extreme observations; the lack of fit was also obvious from the residual plot. The Q-Q (quantile-quantile) plot in Figure 2b shows prevailing coincidence of studentized residuals with a standard normally distributed variable. From the $\mathrm{Q}-\mathrm{Q}$ plots of studentized residuals, it was concluded that the normal assumption made for the full model was generally fulfilled, such that the GBLUP approach was suitable to explore genetic effects on metabolites.

\section{Genetic Variability}

As the estimated genetic variance components cannot be compared among metabolites when expressed on the original scale of $\log _{2}$ intensities, the proportion of genetic variation to the total phenotypic variance $\left(h^{2}\right.$ or $H^{2}$ ), which is a universal measure, is emphasized below. Estimates of variances and heritability based on the full model of all 190 metabolites can be found in Supplemental Figure S2 (available online at http://www.journalof dairyscience.org/). Estimates of $H^{2}$ varied from zero to 0.699 , with a median of 0.125 (standard errors ranged from zero to 0.196 ), and $h^{2}$ varied from zero to 0.569 , with a median of 0.063 (standard errors ranged from zero to 0.117 ). To give an overview of substance groups present in the data, results were sorted according to the 51 identified chemical groups of metabolites (refer-



Butanoic acid, 3-hydroxy-

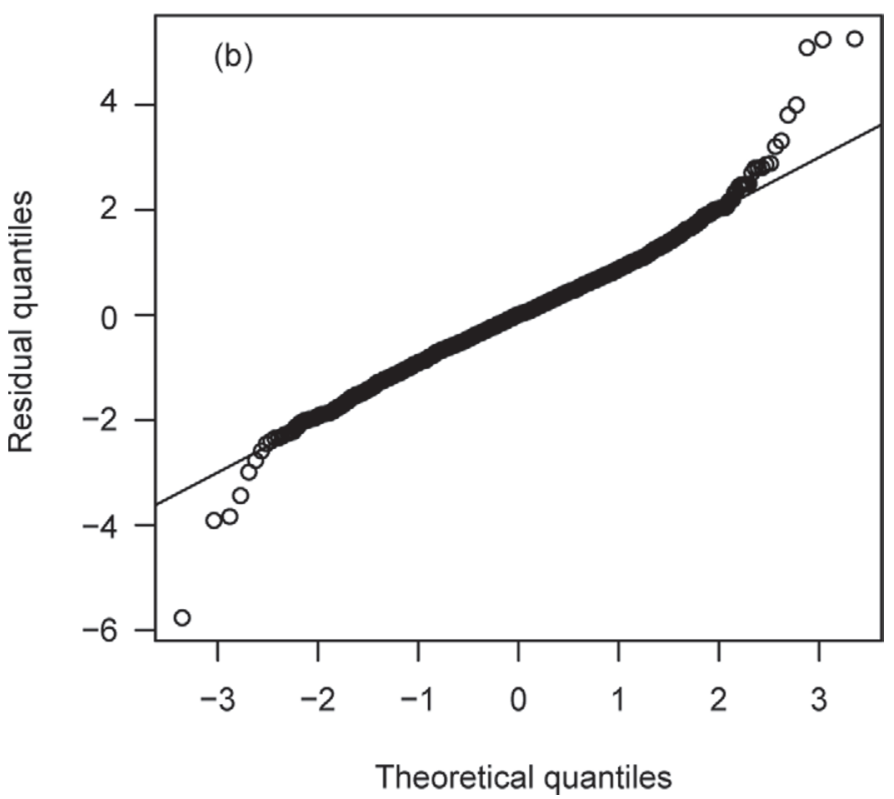

Figure 2. (a) Histogram of $\log _{2}$-transformed metabolite intensities of 3-hydroxybutanoic acid; (b) Q-Q (quantil-quantile) plot of studentized residuals. Dashed line in (a) corresponds to density function of normal distribution with mean $(\mu)$ and variance $\left(\sigma^{2}\right)$ estimated empirically.

ring to GMD; see Supplemental Figure S3, available online at http://www.journalofdairyscience.org/). A shortened list, where metabolite groups are assembled into 17 superordinate chemical groups, is presented in Table 1. This table illustrates the effect of dominance on some metabolites; for example, AA, alcohols, and lactams differed little in terms of estimated $h^{2}$ but $H^{2}$ clearly showed the contribution of dominance variance to alcohols $\left(h^{2}=0.141, H^{2}=0,251\right)$, lactams $\left(h^{2}=\right.$ 
Table 1. Average estimated heritabilities and their interquartile range within superordinate metabolite groups ${ }^{1}$

\begin{tabular}{llcccc}
\hline Group & Group size & $h^{2}$ & IQR $\left(h^{2}\right)$ & $H^{2}$ & IQR $\left(H^{2}\right)$ \\
\hline Alcohol & 5 & 0.141 & 0.201 & 0.251 & 0.328 \\
Aldehyde & 1 & 0.024 & - & 0.081 & - \\
Amine & 3 & 0.096 & 0.178 & 0.295 & 0.032 \\
Amino acid & 18 & 0.154 & 0.173 & 0.171 & 0.187 \\
Carboxylic acid & 19 & 0.121 & 0.138 & 0.197 & 0.133 \\
Conjugate & 2 & 0.048 & 0.035 & 0.101 & 0.140 \\
Indole & 1 & 0.107 & - & 0.150 & - \\
Lactam & 3 & 0.136 & 0.252 & 0.291 & 0.182 \\
Nucleoside & 3 & 0.030 & 0.083 & 0.038 & 0.081 \\
Nucleotide & 2 & 0.000 & 0.000 & 0.055 & 0.110 \\
Other acid & 16 & 0.155 & 0.234 & 0.185 & 0.263 \\
Polyol & 5 & 0.157 & 0.184 & 0.222 & 0.292 \\
Purine & 2 & 0.019 & 0.038 & 0.043 & 0.002 \\
Pyrimidine & 4 & 0.079 & 0.072 & 0.139 & 0.170 \\
Sugar & 18 & 0.161 & 0.288 & 0.197 & 0.340 \\
Terpenoid & 1 & 0.000 & - & 0.006 & - \\
Unspecified $^{2}$ & 87 & 0.088 & 0.125 & 0.144 & 0.165 \\
\hline
\end{tabular}

${ }^{1} h^{2}=$ narrow-sense heritability, $H^{2}=$ broad-sense heritability, IQR = interquartile range.

${ }^{2}$ Unspecified denotes metabolites that could not be assigned to a chemical group.

$\left.0.136, H^{2}=0.291\right)$, and, to a smaller extent, to AA $\left(h^{2}\right.$ $=0.154, H^{2}=0.171$ ), The boxplot of $H^{2}$ in Figure 3a shows the range of $H^{2}$ estimates within each metabolite group. The interquartile range of $H^{2}$ estimates varied strongly between zero and 0.340; for instance, interquartile range was 0.133 for carboxylic acids and 0.328 for alcohols (Table 1).

Significant additive genetic variation was found in 55 of 190 metabolites, meaning that $\sigma_{A}^{2}$ or $\sigma_{p}^{2}$ were greater than zero. False discovery rate-corrected $P$-values are given in Supplemental Figure S2 (available online at http://www.journalofdairyscience.org/). Significant results were mainly found in sugars (6), amino (10), carboxylic (6), and other acids (8). Significant dominance variation was detected only at a metabolite-wise level; for example, in 2-oxoglutaric acid or benzoic acid. In such cases, 37.6 to $100 \%$ of genetic variation was due to dominance (with a moderate level of residual variance). Variation of dominance or polygenic effects was not significantly present at a false discovery rate level. Figure $3 \mathrm{~b}$, which compares estimates of $h^{2}$ and $H^{2}$, also represents these type-III testing results. Significant contributions of additive genetic variation to phenotypic variation were observed for highly heritable metabolites.

The average computing time was $33 \mathrm{~min}$ for the full model, including the $F$-test, but 5 min were required for the reduced model under $H_{0}: \sigma_{p}^{2}=0$ (on a $2.93-\mathrm{GHz}$ multi-user system). Therefore, checking the predictive ability concentrated on the reduced model. Accuracy of genetic value prediction was modest for 3-(4-hydroxyphenyl)-lactic acid $(\rho=0.28)$, glycerol3 -phosphate $(\rho=0.21)$, and ribulose-5-phosphate $(\rho=$ 0.19 ), but low for the other selected metabolites (Table
2). Accuracy of phenotype prediction varied to a lesser extent, with correlations ranging from 0.67 to 0.77 . Further, the mean squared error of prediction showed that lowly heritable metabolites were as good as highly heritable metabolites for predicting total metabolite intensities.

\section{Fixed Effects}

The outcome for testing the effects of farm $\times$ sampling date and measurement day was homogeneous - both effects were significantly present for all metabolites.

In total, 154 of 190 metabolites were significantly affected by lactation day. For 100 metabolites, $p_{e}=2$, meaning that a linear regression was sufficient; for 27 metabolites, $p_{e}=3$, arguing in favor of an additional quadratic term. Hence, higher order regression was only suitable for a minority of the metabolites. Mostly, the precorrected metabolite measurements appeared rather flat over the lactation period. Figure 4 gives 2 arbitrary examples, for which the quadratic regression on lactation day was appropriate despite the flat or almost linear behavior of the median of precorrected metabolites over the lactation period, which was divided into 5 -d intervals.

\section{DISCUSSION}

Milk metabolites are measurable using a GC-MS approach; they represent a new class of milk traits with unknown genetic background. Before investigating relationships of milk metabolites to classical milk traitsto performance or functional traits - it is necessary to characterize their genetic basis. The current study tackled this question, focusing on heritability and mode of 

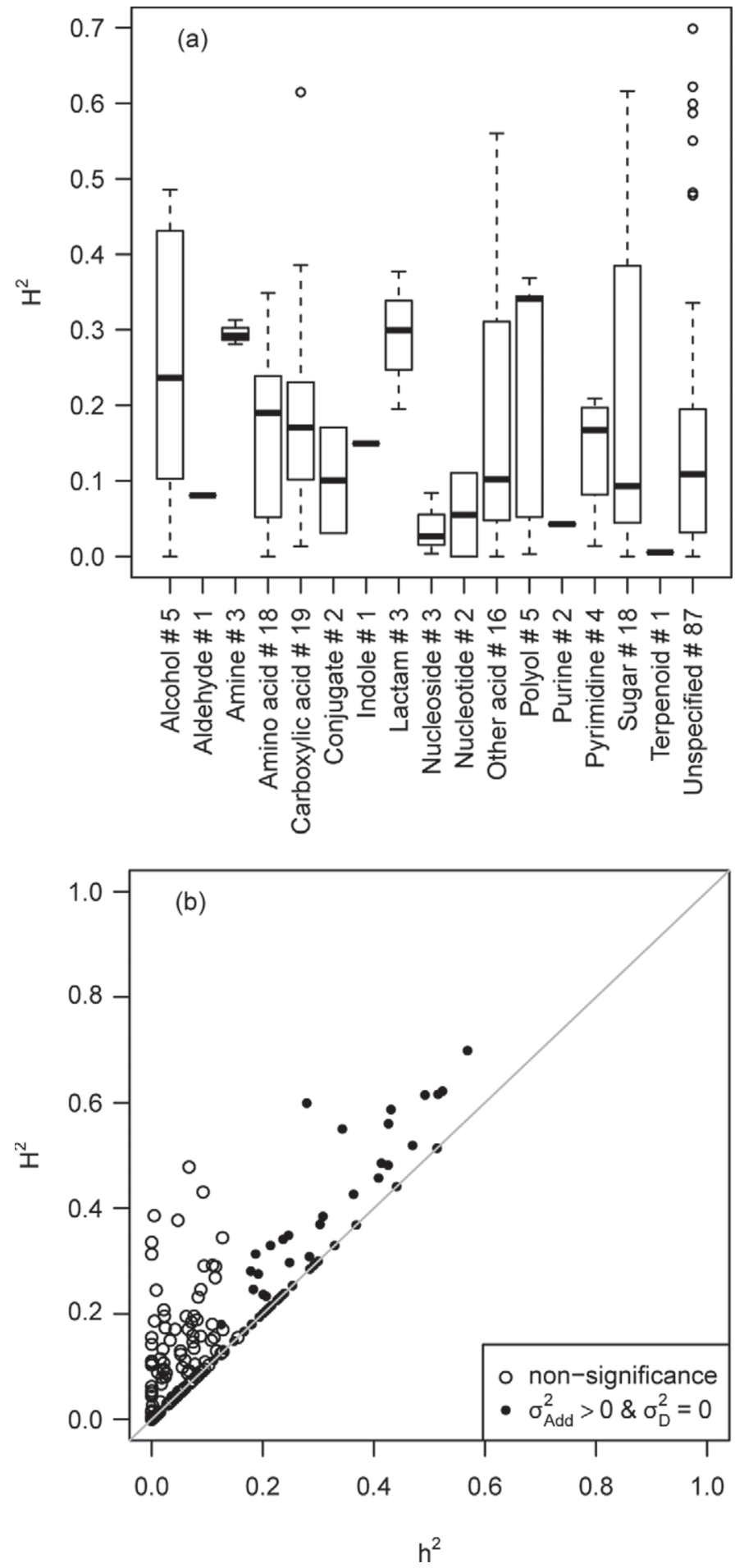

Figure 3. (a) Boxplot of broad-sense heritability within groups of metabolites; (b) comparison of narrow-sense heritability $h^{2}$ versus broad-sense heritability $H^{2}$. Variance components: additive genetic variance $\sigma_{\text {Add }}^{2}$, which comprises marker-based $\left(\sigma_{A}^{2}\right)$ and polygenic $\left(\sigma_{p}^{2}\right)$ variance, dominance variance $\sigma_{D}^{2}$. "Unspecified" denotes metabolites that could not be assigned to a chemical group. inheritance for these new molecular milk composition traits and their suitability for genetic evaluations.

\section{Genetic Variability}

The genomic relationship between animals was used to model the covariance structure for additive and dominance genetic values. Residual additive genetic variation not captured by the SNP markers was modeled using the pedigree relationship. Heritabilities for the investigated milk metabolites in the sample of commercial dairy cows were mostly small to intermediate; half the metabolites had broad-sense heritability estimates smaller than 0.125 . Almost $30 \%$ of the metabolites showed significant contribution of additive genetic variation to the phenotypic variation, but dominance variance was not significantly present. When the full model presented in this paper is compared with a typical animal model (including only pedigree-based genetic effects), then additional additive genetic variation could be explained by markers (Figure 5a). The average ratio of $h^{2}$ estimated with both models was 1.689. In contrast to the animal model, the full model considered realized relationship among animals and therefore also accounted for Mendelian sampling effects. Thus, as expected, the increase in heritability also went along with a prevailingly reduced standard error of $h^{2}$ estimates (Figure 5b). For 97 of 138 metabolites, a lower standard error was observed; for the remaining 52 metabolites, the standard error was not estimable because the polygenic variance was estimated close to zero in the animal model.

The few examples studied for predictive ability showed rather limited power to predict genetic values; the accuracy of genetic value prediction was at most 0.28 , despite $H^{2}=0.584$ (Table 2). For comparison, the expected accuracy $(r)$ was approximated according to Daetwyler et al. (2010) using an effective number of chromosome segments $M_{e}=2 N_{e} L$ with effective population size $N_{e}=100$ and chromosome length $L=30 \mathrm{M}$. For the extreme examples, $r=0.33\left(H^{2}=0.584\right)$ and $r=0.23\left(H^{2}=0.263\right)$. These values were higher than the observed ones, which might be due to, among other reasons, an overestimated heritability or misspecified $M_{e}$. The accuracy of phenotype prediction was more convincing for the selected metabolites; the minimum correlation was 0.67 . This outcome implied that more than $80 \%$ of the variation of metabolites could be explained by linear relationships using GBLUP, leaving a minor part unexplained.

A conceivable approach to increase predictive ability is combining selected metabolites with performance traits with which they are highly correlated in multivariate investigations. Therein, genetic correlations 
Table 2. Predictive ability of the genomic BLUP approach for selected metabolites via leave-one-out crossvalidation $^{1}$

\begin{tabular}{lcccc}
\hline Metabolite & $H^{2}(\mathrm{SE})$ & $\operatorname{cor}\left(y, \hat{u}_{a}+\hat{u}_{d}\right)$ & $\operatorname{cor}(y, \hat{y})$ & $\operatorname{MSE}$ \\
\hline Lactic acid, 3-(4-hydroxyphenyl)- & $0.584(0.122)$ & 0.284 & 0.700 & 0.599 \\
Glycerol-3-phosphate & $0.485(0.129)$ & 0.209 & 0.665 & 0.325 \\
Ribulose-5-phosphate & $0.484(0.137)$ & 0.185 & 0.709 & 1.036 \\
Glucose, 2-amino-2-deoxy- & $0.321(0.147)$ & 0.092 & 0.668 & 0.586 \\
Glucose, 1,6-anhydro, $\beta$ - & $0.263(0.172)$ & 0.013 & 0.769 & 0.663 \\
\hline
\end{tabular}

${ }^{1}$ Parameters: broad-sense heritability $H^{2}$ based on the reduced model (polygenic variance $\sigma_{p}^{2}=0$ ) and its SE; observed $(y)$ and predicted $(\hat{y})$ metabolite intensity, predicted additive genetic value $\hat{u}_{a}$ and predicted dominance genetic value $\hat{u}_{d}$; mean squared error (MSE) of prediction: MSE $=\frac{1}{n} \sum_{i=1}^{n}\left(y_{i}-\hat{y}_{i}\right)^{2}$. Here, $i$ denotes the sample number and $n$ the total number of samples.

between multiple traits and phenotypic correlations are considered, because metabolites and milk traits are likely measured from the same milk sample. This issue requires a large sample size to properly estimate correlations, but will lead to an improved genetic value prediction of metabolites.

Milk metabolites, in general, might not be suitable for breeding purposes, but many metabolites showed significant genetic variability. Some of those components may be used to permanently indicate health risks, such as mastitis, but a permanent relation to diseases remains to be verified at different lactation stages. Davis et al. (2004) analyzed the relationship between mastitis and the concentration of lactic acid; as heritability of lactic acid was estimated at a low level in the sample data (i.e., $H^{2}=0.013$ ), this outcome suggests that lactic acid can be better seen as a temporary indicator for mastitis than as a permanent indicator. Metabolites that mostly reflect environmental or temporal variable status (as lactic acid does) may be useful to indicate variable disorders, and they might gain practical importance for monitoring and other management purposes (Melzer et al., 2013).

To improve the robustness of cows to metabolic disorders through specific breeding programs, concentration of selected metabolites with proved relation to a disease could be measured regularly during milk performance tests, for example, via infrared spectroscopy - as it is done for milk performance traits. This will also lead to a large collection of samples, thereby improving the predictive ability of metabolites. For instance, assuming $H^{2}=0.03$, about 6,700 individual observations are required to achieve an expected accuracy of 0.5.

Investigations incorporated SNP with minor allele frequency $\geq 5 \%$. This implied that $\mathbf{G}_{a}$ and $\mathbf{G}_{d}$ were not positive definite as required for solving the mixed model equations. The ASReml options were used to regularize these covariance matrices; ASReml formed an expanded singular representation of the corresponding inverse matrices (Gilmour et al., 2008) and the outcome was similar to that of a regularization method, where a small value $\varepsilon$ was added to the diagonal elements of the respective covariance matrix. As an alternative, one could select SNP with minor allele frequency $\geq 10 \%$. In test runs, identical results were observed when adding $\varepsilon=0.001$ to the diagonal; other results were similar in terms of estimated heritabilities.

\section{Fixed Effects}

Similar to a test-day model (Ptak and Schaeffer, 1993), some fixed effects on milk metabolite intensities were considered. Due to the large amount of data, effects of farm and sampling date were considered to be cross-classified. As all cows on a specific farm were fed the same food, an effect of diet was also considered by the effect of farm $\times$ sampling date. Further, linear and quadratic regressions on lactation day were considered to account for a variable metabolite state between $d$ 21 and 120 of lactation. Outside this lactation period a stronger deviation of metabolite intensities might be observed among animals requiring higher order regression. This study concentrated on the limited lactation period, assuming that the metabolism is mainly stable and not affected by metabolic diseases often appearing at early lactation (Goff and Horst, 1997). As milk metabolites were analyzed, the test-day model was extended to include the effect of measurement day, which accounts for batch effects mainly due to detector sensitivity when measuring the molecule intensities via MS (Steinfath et al., 2008).

\section{Different Types of Metabolic Substances}

According to the public database GMD, the metabolites could be assigned to 51 chemical groups. The AA represented the main group in the sample data, comprising 12 proteinogenic (e.g., alanine) and 6 nonproteinogenic (e.g., sarcosine) substances. The next frequently allocated groups were hydroxy (6) and dicarboxylic 
(a)

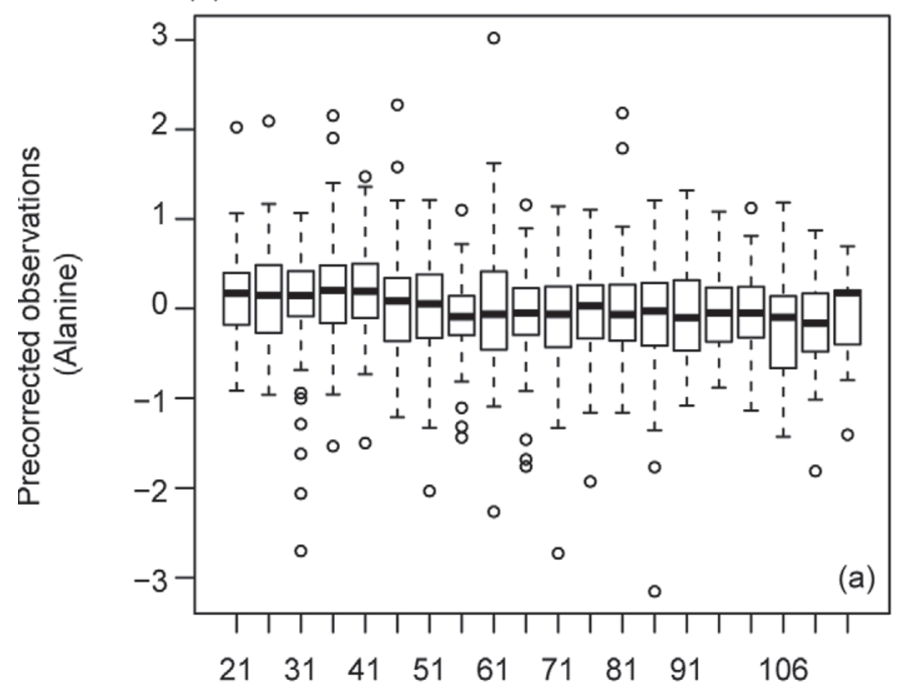

(b)

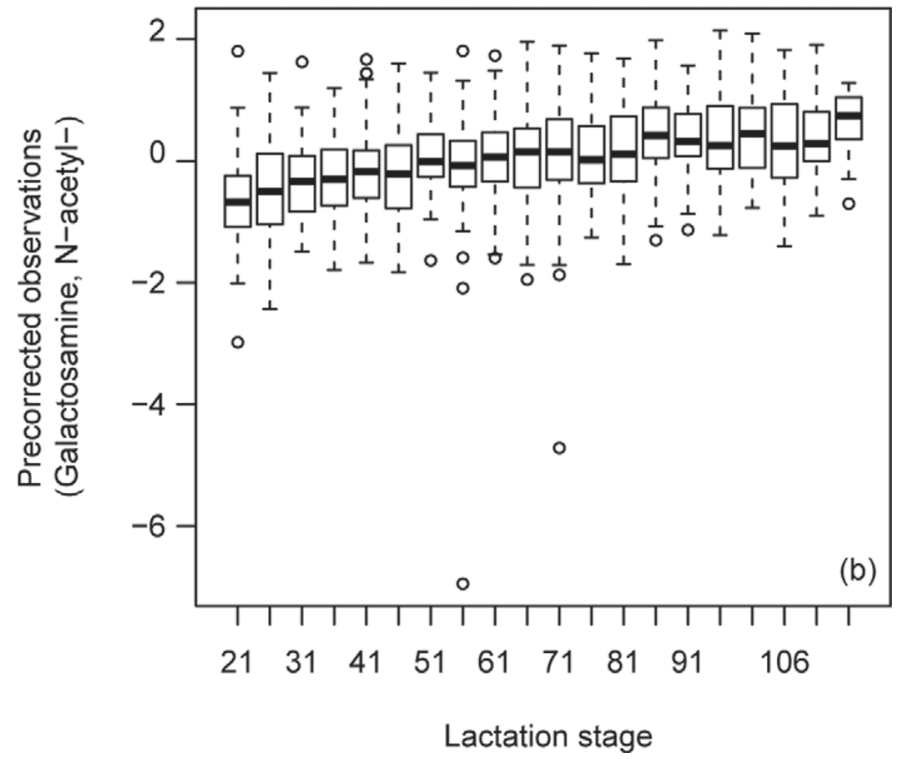

Figure 4. Boxplot for precorrected metabolite measurements depending on lactation interval: (a) alanine and (b) $N$-acetylgalactosamine. Lactation period was divided into 5-d intervals. Precorrection was obtained via linear mixed model including fixed effects of farm $\times$ sampling date and measurement day and a random sire effect.

acids (6; see Supplemental Figure S3, available online at http://www.journalofdairyscience.org/). In the shortened presentation of results (Table 1), where the estimates referred to superordinate metabolite groups, di- and tricarboxylic, aromatic, hydroxyl, and oxoacids were collected into carboxylic acids. Furthermore, all sugars and their derivatives, as well as modified sugars, were subsumed to sugars in general.
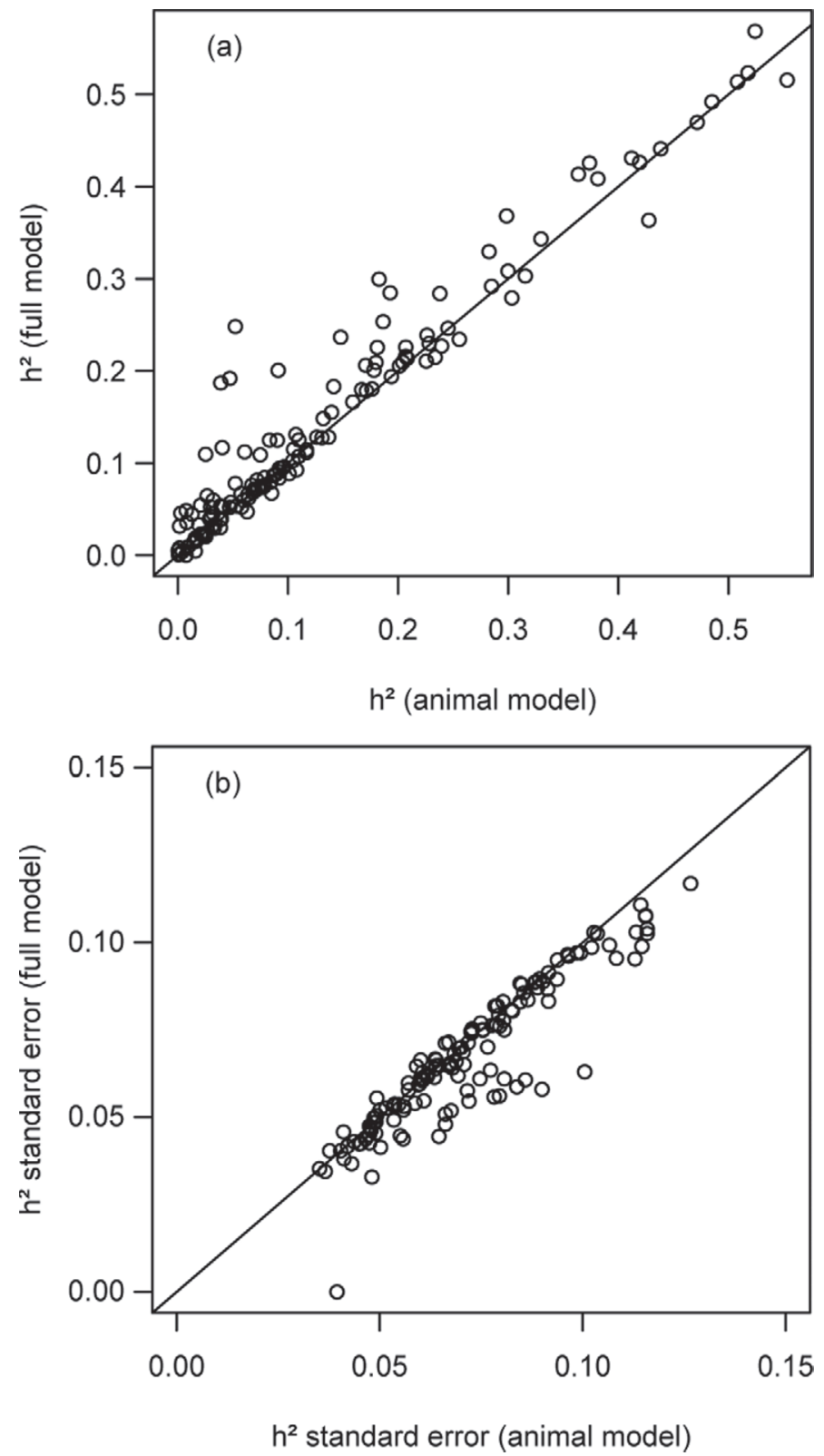

Figure 5. (a) Narrow-sense heritability based on full model (additive and dominance variance captured by markers and polygenic variance) versus heritability based on animal model (polygenic variance); (b) standard error of heritability estimates via full model versus animal model.

Compared with classical milk traits of the same animal sample (Melzer et al., 2013), such as content of protein $\left(h^{2}=0.309\right)$, fat $\left(h^{2}=0.227\right)$, or lactose $\left(h^{2}=\right.$ $0.136)$, heritabilities of milk metabolites were mostly below these reference values in the population studied. A few metabolites show larger estimates; the maximum value was estimated for 3-(4-hydroxyphenyl)-lactic acid at $h^{2}=0.569$. Amino acids are mainly associated with 
protein production and breakdown, and on average $h^{2}=0.154$ in this chemical group (Table 1). For FA components, which can be mainly found in the group of carboxylic acids, it was estimated that $h^{2}=0.121$. In the group of sugars, $h^{2}=0.161$. In each group, the estimated $h^{2}$ strongly varied as it was also observed for $H^{2}$ (Figure 3a).

\section{Pathways}

To find reasons for the wide range of estimated heritabilities, functional associations between metabolites were investigated exemplarily using the KEGG database (http://www.genome.jp/kegg). A main metabolic pathway, glycolysis, converts glucose into pyruvates releasing energy in form of ATP. This process includes, among others, the following metabolites that were identified in the data: 2-phosphoglyceric acid $\left(H^{2}=\right.$ 0.046), 3-phosphoglyceric acid $\left(H^{2}=0.308\right)$, phosphoenolpyruvic acid $\left(H^{2}=0.441\right)$, and pyruvic acid $\left(H^{2}\right.$ $=0.155)$. Even though these components are closely functionally related in glycolysis, heritability strongly differed between them. Metabolites were positively correlated in this subset; correlations between residuals obtained from the full model varied from 0.064 to 0.84 . Thus, the $\log _{2}$-transformed sum of (raw) metabolite intensities identified in glycolysis was investigated, and the genetic variability was determined based on the full model. For the derived variable, $H^{2}=0.238$, which coincided roughly with the average heritability of single metabolites.

In the pentose phosphate pathway, a few highly heritable metabolites were recognized: fructose-6-phosphate $\left(H^{2}=0.686\right)$, 6-phosphogluconic acid $\left(H^{2}=0.426\right)$, and ribulose-5-phosphate $\left(H^{2}=0.519\right)$. Some metabolites with small to intermediate heritability were also recognized: gluconic acid $\left(H^{2}=0.297\right)$, pyruvic acid, and 3-phosphoglyceric acid. Except gluconic acid, all residual metabolite intensities were positively correlated; correlations ranged from 0.045 to 0.84 . The $\log _{2^{-}}$ transformed sum of positively correlated metabolite measurements resulted in $H^{2}=0.425$. This condition suggests that at least parts of the energy metabolism might be affected by genetics.

As some metabolites contribute to different pathways (for instance, pyruvic acid participates in glycolysis and pentose phosphate pathway), it might be an interesting point to further explore the relationship between pathways and their genetic characteristic. Eventually, metabolic flux modes (Hoffmann et al., 2006), which could be understood as realized paths of metabolite conversion, might show more elevated levels of genetic determination than the single metabolite intensities of the present study.

\section{Favorable Metabolites}

Milk and its metabolic products, such as BMO or vitamins, are important for human nutrition. The sample data included few metabolites that contribute to vitamins: pantothenic acid $\left(\mathrm{B}_{5}\right)$, pyridoxal and pyridoxamine $\left(\mathrm{B}_{6}\right)$, as well as one component which is related to thiazol $\left(\mathrm{B}_{1}\right)$, 4-methyl-5-hydroxyethyl-thiazol. These components seem to be affected more by management (e.g., feed and environment), than by genetics. Intermediate heritability was, however, found for pyridoxal $\left(H^{2}=0.233\right)$, and 4-methyl-5-hydroxyethyl-thiazol $\left(H^{2}\right.$ $=0.226$; see Supplemental Figure S2, available online at http://www.journalofdairyscience.org/).

Among all metabolites, 3 main substances were found that contribute to the possible structures identified for BMO [Tao et al., 2008; i.e., $N$-acetylneuraminic acid (Neu5Ac), $N$-acetylgalactosamine (GalNAc), and $N$-acetylglucosamine (GlcNAc)]. As the concentration of beneficial oligosaccharides is lower in bovine than human milk, extraction of BMO from dairy sources is useful to enrich, for instance, infant formula (Zivkovic and Barile, 2011). The absolute quantity of substances found in the sample could not be determined, however, because a calibration for metabolite intensities in milk was not implemented in this study. Martín et al. (2001) reported that sialic acids, with their predominant component, Neu5Ac, amount to approximately $170 \mathrm{mg} / \mathrm{kg}$ of mature milk. For comparison, the BMO components glucose and galactose, which were not measured in this study, contribute up to 60 and $20 \mathrm{mg}$ per $\mathrm{kg}$, respectively (Töpel, 2004). Two of the metabolites, GalNAc and GlcNAc, had high estimates of heritability, $H^{2}=$ 0.587 and $H^{2}=0.482$, respectively (see Supplemental Figure S2, available online at http://www.journalof dairyscience.org/). Food producers may desire milk of daughters of bulls with extraordinarily high breeding value with respect to favorable substances on special farms. The total oligosaccharide intensity is assumed to decrease from colostrum to later lactation milk (Tao et al., 2009). Colostrum was not available in this study, but GalNAc and GlcNAc increased slightly over the different stages of lactation (Figure 4b), and Neu5Ac was at least constant until d 100. Future studies may investigate which period of lactation BMO abundance is most suitable for extraction.

\section{CONCLUSIONS}

In our study of the genetic background of 190 milk metabolites, heritability was found at a low to intermediate level. Even though the sample size of our experiment was small $(n=1,295)$, results indicate the mode of inheritance. Most genetic variability was explained by additive genetic sources comprising pedi- 
gree- and marker-based genetic variance. Significant additive genetic variation was found in 55 metabolites. Dominance variance was not significantly present. The few highly heritable metabolites studied for predictive ability showed modest to low accuracy of genetic value prediction. At the same time, accuracy of predicting total metabolite intensities was at a high level, confirming the general suitability of the GBLUP approach for studying variation of metabolite measurements. Future research should address the identification of locus-specific genetic effects on selected metabolites. This could be achieved by simultaneously estimating SNP effects over the whole genome (e.g., via BayesB; Meuwissen et al., 2001), instead of studying total genetic values, as was done in the current study. Relations of milk metabolite profiles to standard milk performance test traits should also be further investigated to discover whether it will be possible to elucidate the molecular basis of these classical traits using new, detailed milk composition traits.

\section{ACKNOWLEDGMENTS}

This study was part of the FUGATO plus project "Bovine Integrative Bioinformatics for Genomic Selection" with financial support of the German Federal Ministry of Education and Research (BMBF). The authors thank the participating herd owners and cooperation partners. The contribution of $\ddot{A}$. Eckardt (Max Planck Institute for Molecular Plant Physiology, Potsdam-Golm, Germany), who implemented GC-MS technology for milk samples and measured the metabolite profiles, is acknowledged. The SNP genotypes were measured by the working group of T. Meitinger and P. Lichtner (Helmholtz Zentrum München, Germany). S. Jakubowski, S. Hartwig, and S. Wolf (LKV Güstrow, Germany) provided milk performance data and contributed to discussions during the project. We acknowledge the support of F. Reinhardt (vit Verden), who assembled the pedigree data. Special thanks are given to colleagues at the Leibniz Institute for Farm Animal Biology (Dummerstorf, Germany): R. Grahl, who collected blood and milk samples; C. Reiko, who helped to prepare blood samples for DNA extraction; the working group of J. Vanselow, who provided technical facilities for DNA preparation; A. Rief, who built the SNP marker map; C. Kühn, who gave valuable remarks; and especially M. Nimz, M. Anders, and M. Spitschak, who gave assistance in preparation. We also thank the anonymous reviewers for their comments.

\section{REFERENCES}

Altschul, S. F., W. Gish, W. Miller, E. W. Myers, and D. J. Lipman. 1990. Basic local alignment search tool. J. Mol. Biol. 215:403-410.
Benjamini, Y., and Y. Hochberg. 1995. Controlling the false discovery rate: A practical and powerful approach to multiple testing. J. R. Stat. Soc., B 57:289-300.

Browning, S. R., and B. L. Browning. 2007. Rapid and accurate haplotype phasing and missing-data inference for whole-genome association studies by use of localized haplotype clustering. Am. J. Hum. Genet. 81:1084-1097.

Calus, M. P. L., H. A. Mulder, S. McParland, E. Strandberg, E. Wall, and J. W. M. Bastiaansen. 2011. Identification of Mendelian inconsistencies between SNP and pedigree information of sibs. Genet. Sel. Evol. 43:34.

Cuadros-Inostroza, A., C. Caldana, H. Redestig, M. Kusano, J. Lisec, H. Pena-Cortes, L. Willmitzer, and M. A. Hannah. 2009. Targetsearch-A Bioconductor package for the efficient preprocessing of GC-MS metabolite profiling data. BMC Bioinformatics 10:428.

Daetwyler, H. D., R. Pong-Wong, B. Villanueva, and J. A. Woolliams. 2010. The impact of genetic architecture on genome-wide evaluation methods. Genetics 185:1021-1031.

Davis, S. R., V. C. Farr, C. G. Prosser, G. D. Nicholas, S.-A. Turner, J. Lee, and A. L. Hart. 2004. Milk L-lactate concentration is increased during mastitis. J. Dairy Res. 71:175-181.

Geishauser, T., K. Leslie, J. Tenhag, and A. Bashiri. 2000. Evaluation of eight cow-side ketone tests in milk for detection of subclinical ketosis in dairy cows. J. Dairy Sci. 83:296-299.

Gilmour, A., B. Gogel, B. Cullis, and R. Thompson. 2008. ASReml User Guide Release 3.0. VSN International Ltd., Hemel Hempstead, UK.

Goff, J. P., and R. Horst. 1997. Physiological changes at parturition and their relationship to metabolic disorders. J. Dairy Sci. 80:1260-1268

Habier, D., R. L. Fernando, and J. C. M. Dekkers. 2007. The impact of genetic relationship information on genome-assisted breeding values. Genetics 177:2389-2397.

Hastie, T., R. Tibshirani, and J. Friedman. 2009. The Elements of Statistical Learning: Data Mining, Inference, and Prediction. Springer Verlag, New York, NY.

Hoffmann, S., A. Hoppe, and H.-G. Holzhütter. 2006. Composition of metabolic flux distributions by functionally interpretable minimal flux modes (minmodes). Genome Inform. 17:195-207.

Kenward, M. G., and J. H. Roger. 1997. Small sample inference for fixed effects from restricted maximum likelihood. Biometrics 53:983-997.

Klein, M. S., M. F. Almstetter, G. Schlamberger, N. Nürnberger, K Dettmer, P. J. Oefner, H. H. D. Meyer, S. Wiedemann, and W. Gronwald. 2010. Nuclear magnetic resonance and mass spectrometry-based milk metabolomics in dairy cows during early and late lactation. J. Dairy Sci. 93:1539-1550.

Klein, M. S., N. Buttchereit, S. Miemczyk, A.-K. Immervoll, C. Louis, S. Wiedemann, W. Junge, G. Thaller, P. J. Oefner, and W. Gronwald. 2012. NMR metabolomic analysis of dairy cows reveals milk glycerophosphocholine to phosphocholine ratio as prognostic biomarker for risk of ketosis. J. Proteome Res. 11:1373-1381.

Lisec, J., N. Schauer, J. Kopka, L. Willmitzer, and A. R. Fernie. 2006. Gas chromatography mass spectrometry-based metabolite profiling in plants. Nat. Protoc. 1:387-396.

Littledike, E. T., J. W. Young, and D. C. Beitz. 1981. Common metabolic diseases of cattle: Ketosis, milk fever, grass tetany, and downer cow complex. J. Dairy Sci. 64:1465-1482.

Martín, M.-J., S. Martín-Sosa, L.-A. García-Pardo, and P. Hueso. 2001. Distribution of bovine milk sialoglycoconjugates during lactation. J. Dairy Sci. 84:995-1000.

Melzer, N., S. Jakubowski, S. Hartwig, U. Kesting, S. Wolf, G. Nürnberg, N. Reinsch, and D. Repsilber. 2010. Design, infrastructure and database structure for a study on predicting of milk phenotypes from genome wide snp markers and metabolite profiles. Abstract ID 0427 in Proc. 9th World Congress on Genetics Applied to Livestock Production. German Society for Animal Science, ed. zwonull media GbR, Leipzig, Germany.

Melzer, N., D. Wittenburg, S. Hartwig, S. Jakubowski, U. Kesting, L. Willmitzer, J. Lisec, N. Reinsch, and D. Repsilber. 2013. Investigating associations between milk metabolite profiles and milk 
traits of Holstein cows. J. Dairy Sci. 96:1521-1534. http://dx.doi. org/10.3168/jds.2012-5743.

Meuwissen, T. H., B. J. Hayes, and M. E. Goddard. 2001. Prediction of total genetic value using genome-wide dense marker maps. Genetics 157:1819-1829.

Nicholson, J. K., J. C. Lindon, and E. Holmes. 1999. 'Metabonomics': Understanding the metabolic responses of living systems to pathophysiological stimuli via multivariate statistical analysis of biological NMR spectroscopic data. Xenobiotica 29:1181-1189.

Ptak, E., and L. Schaeffer. 1993. Use of test day yields for genetic evaluation of dairy sires and cows. Livest. Prod. Sci. 34:23-34.

R Development Core Team. 2011. R: A Language and Environment for Statistical Computing. R Foundation for Statistical Computing, Vienna, Austria. http://www.R-project.org/.

Ruppert, D., M. P. Wand, and R. J. Carroll. 2003. Semiparametric Regression. Cambridge University Press, Cambridge, UK.

Rutten, M. J. M., H. Bovenhuis, J. M. L. Heck, and J. A. M. van Arendonk. 2011. Predicting bovine milk protein composition based on Fourier transform infrared spectra. J. Dairy Sci. 94:5683-5690.

Self, S. G., and K.-Y. Liang. 1987. Asymptotic properties of maximum likelihood estimators and likelihood ratio tests under nonstandard conditions. J. Am. Stat. Assoc. 82:605-610.

Soyeurt, H., P. Dardenne, F. Dehareng, G. Lognay, D. Veselko, M. Marlier, C. Bertozzi, P. Mayeres, and N. Gengler. 2006. Estimating fatty acid content in cow milk using mid-infrared spectrometry. J. Dairy Sci. 89:3690-3695.
Steinfath, M., D. Groth, J. Lisec, and J. Selbig. 2008. Metabolite profile analysis: From raw data to regression and classification. Physiol. Plant. 132:150-161.

Tao, N., E. J. DePeters, S. Freeman, J. B. German, R. Grimm, and C. B. Lebrilla. 2008. Bovine milk glycome. J. Dairy Sci. 91:37683778 .

Tao, N., E. J. DePeters, J. B. German, R. Grimm, and C. B. Lebrilla. 2009. Variations in bovine milk oligosaccharides during early and middle lactation stages analyzed by high-performance liquid chromatography-chip/mass spectrometry. J. Dairy Sci. 92:2991-3001.

The Bovine Genome Sequencing and Analysis Consortium. 2009. The genome sequence of taurine cattle: A window to ruminant biology and evolution. Science 324:522-528.

Töpel, A. 2004. Chemie und Physik der Milch: Naturstoff, Rohstoff, Lebensmittel. Behr's Verlag, Hamburg, Germany.

VanRaden, P. M. 2008. Efficient methods to compute genomic predictions. J. Dairy Sci. 91:4414-4423.

Zeng, Z.-B., T. Wang, and W. Zou. 2005. Modeling quantitative trait loci and interpretation of models. Genetics 169:1711-1725.

Ziegler, A., I. R. König, and J. R. Thompson. 2008. Biostatistical aspects of genome-wide association studies. Biom. J. 50:8-28.

Zivkovic, A. M., and D. Barile. 2011. Bovine milk as a source of functional oligosaccharides for improving human health. Adv. Nutr. $2: 284-289$ 